\title{
FLORISTIC WOODY COMPOSITION OF REVEGETATED MINING SITES IN THE BRAZILIAN FEDERAL DISTRICT
}

\author{
COMPOSIÇÃO FLORÍSTICA LENHOSA DE JAZIDAS REVEGETADAS NO \\ DISTRITO FEDERAL
}

\section{Rodrigo Studart CORRÊA ${ }^{1}$; Benício de MÉLO FILHO ${ }^{2}$; Cristiane de Queiroz PINHEIRO ${ }^{3}$; Perseu Fernando dos SANTOS ${ }^{4}$}

1. Docente da Universidade de Brasília - FUP, Brasília, DF, Brasil. rscorrea@unb.br; 2. Engenheiro Florestal - D.Sc. da Rede Brasileira de Recuperação Ambiental, Brasília, DF, Brasil; 3. Engenheira Florestal - M.Sc. do Ministério do Meio Ambiente, dos Recursos Hídricos e da Amazônia Legal, Brasília, DF, Brasil; 4. Docente da Universidade Católica de Brasília - UCB, Brasília, DF, Brasil.

\begin{abstract}
The extension degraded by mining in the Brazilian Federal District - BFD is proportionately five times larger than country's average. The restoration of plant communities at these sites is ecologically necessary and legally compulsory. Native woody species are commonly used for ecosystem restoration and this study aimed to identify the floristic composition of woody species introduced in BDF mining sites and discuss its outcomes. The floristic survey was conducted by sampling groups of one hundred individuals until sampling sufficiency was achieved. In total 4,500 plants were sampled in ten sites, which housed 92 autochthonous and 21 allochthonous woody species. Plant communities in the sites assembled between 13 and 62 species, planted at low density - $467 \pm 222$ plants ha ${ }^{-1}$. The preferential use of autochthonous species in the revegetation projects resembles the standards recommended by successional restoration models, but the floristic diversity and plant density in initial communities fall below the values deemed as ideal. The 92 autochthonous species include representatives of various habitats, ecological groups and dispersal syndromes. These 92 species currently in use may be regrouped in initial plant communities denser and more diverse than those found in the revegetated sites. The surveyed plant communities were massively composed of arboreal species and such pattern may drive succession towards the formation of forest ecosystems where previously inhabited savanna formations.
\end{abstract}

KEYWORDS: Ecological restoration. Revegetation. Mining areas. Brazilian savanna.

\section{INTRODUCTION}

The Cerrado is the second largest Brazilian biome, occurs in fourteen out 26 states, occupies nearly $25 \%$ of the national territory and houses over twelve thousand species of vascular plants (MENDONÇA et al., 2008a). This biome consists of a mosaic of grassland, savanna and forest formations, which confers upon it a high diversity of habitats and the richest savanna in plant species in the world (MENDONÇA et al., 1998).

Studies report that agriculture, livestock breeding, urbanization and mining are responsible for the loss of more $50 \%$ of Cerrado native vegetation coverage (KLINK; MACHADO, 2005; MENDONÇA et al., 2008a). High degree of endemism, high biodiversity and significant human impacts on natural ecosystems have ranked the Cerrado biome amongst the 34 hotspots for global biodiversity conservation (IUCN, 2010). The Brazilian Federal District - BFD is located on the central part of Cerrado, where conflicts between conservation measures and economic activities are intense. In addition to the damage caused by agriculture and urbanization, almost $1 \%$ of the BFD's territory is taken by mines of clay, gravel, sand and limestone, a percentage five times higher than national average (CORRÊA et al., 2004; STARR et al., 2013).

The destruction of ecosystems by human activities has placed a number of species at risk of extinction. Conservationist plans recommend habitat restoration as a means of offsetting biodiversity losses (MARON; HOBBS, 2012), increasing carrying capacity, and lowering extinction risk (ANAND; DESROCHERS, 2004). The use of seedlings and seeds is the most viable alternative for the restoration of ecosystems that have lost most of resilience, like areas degraded by mining (DURIGAN, 2003; SILVA; CORRÊA, 2008). This practice began in Brazil almost two centuries ago (MARTINS, 1966), and the current use of native species in successional models aims to shorten the stages of natural succession by establishing trees in degraded areas that would be spontaneously colonized by herbs in the early stages of ecological succession (BARBOSA et al., 2003; REIS et. al., 2003; MARTINS et al., 2012).

The revegetation of a degraded area is currently considered the beginning of an ecosystem restoration, since ecological succession is the main course of restoration in tropical regions (CORLETT; HAU, 2000). Restoration projects aim to recover damaged ecosystems by creating conditions that will 
prompt communities to follow their natural pathways where ecological processes were destroyed (ANAND; DESROCHERS, 2004; SER, 2004). In this sense the recovery of biomass and biodiversity is considered necessary to restore and maintain ecosystem functioning (LYONS et al., 2005; MOKANY et al., 2008). Techniques involving facilitation, nucleation, keystone and umbrella species, and others have more recently been incorporated into successional restoration models (REIS et al., 2003; LYONS et al., 2005).

Tree plantation is the oldest and most common practice used for rehabilitation of lands degraded by human activities because trees provide a pronounced visual effect on the landscape (SINGH et al., 2002; RUIZ-JAEN; AIDE, 2005; SILVA; CORREAA, 2008). Trees and shrubs also play a fundamental role in plant recruitment and ecological succession by increasing ecological structure and providing perches and food for fauna (CORLETT; HAU, 2000; KAGEYAMA et al., 2003; RUIZ-JAEN; AIDE, 2005; BARBOSA; PIZO, 2006). The appropriate assembly of plant species that will compose initial communities in degraded areas is essential for the success of restoration projects (MELO et al., 2004; STARR et al., 2013). In such view, this study aimed to identify the woody species assembled as initial plant communities in sites previously degraded by mining activities in the Brazilian Federal District - BFD and discuss outcomes of the floristic composition adopted.

\section{MATERIAL AND METHODS}

\section{Study Area}

The study was conducted in the Federal District of Brazil - BFD, which occupies $5,814 \mathrm{~km}^{2}$ of the Brazilian Central Plateau. The region's topography varies from flat to gently slope, with average altitude of $1,100 \mathrm{~m}$. Climate is Tropical of Savanna (Aw - Köppen-Geiger) with well-defined wet and dry seasons (PEEL et al., 2007), annual mean temperature of $21-24 \circ \mathrm{C}$, and annual rainfall ranges from 1,200 to $1,600 \mathrm{~mm}, 84 \%$ of which precipitate in summer (INMET, 2012).

\section{Floristic survey}

Planted woody species were sampled in revegetated mining sites that were subject to restoration projects, namely the recovery of degraded ecosystems as close as possible to their original condition (Brazilian Federal Law Nr. 9.985/2000). All study sites were located in areas originally covered by savannic formations on either Oxisol or Inceptisol soils.

Sampling works in each site began soon after revegetation works, which occurred from 2005 to 2010. Based on Equations 1 and 2 (SNEDECOR; COCHRAN, 1989), four hundred plants were initially sampled in each site and then checked for sampling sufficiency through species rarefaction curves (GOTELLI; COLWELL, 2001). Sampling works were extended wherever necessary until sampling sufficiency was achieved in each revegetated site, which required the sampling of 400 to 900 individuals in each of the ten studied locations (Table 1).

$$
\begin{aligned}
& \mathrm{n}_{0}=\frac{1}{E^{2}} \Rightarrow \frac{1}{0.05^{2}}=400 \text { individuals } \quad \text { (Equation 1) } \\
& \mathrm{n}=\frac{N \times n_{o}}{N+n_{o}} \\
& \text { where, } \\
& \mathrm{n}_{0}=\text { sample size for an infinite size } \\
& \text { population, at 5\% confidential level } \\
& \mathrm{n}=\text { sample size for a known size population } \\
& \mathrm{N}=\text { population size }
\end{aligned}
$$

The floristic survey was done through traversal shaped scanning (PINHEIRO et al., 2009), using at least four sample groups of one hundred planted woody individuals each one (Equation 1). The starting point of each sample group was selected at random, and covered up a rectangle area from that point on until one hundred plants were sampled. The area occupied by every group of one hundred planted individuals was used for the estimation of planting density in each revegetated site. Part of plants from species not identified in situ were collected and pressed for subsequent identification through literature and comparison with exsiccates deposited in the University of Brasilia's herbarium. Taxonomic classification was based on The Angiosperm Phylogeny Group system (APG III, 2009), and taxa names were updated as per the nomenclature of the Missouri Botanical Garden (MOBOT, 2012).

Ten revegetated mining sites were sampled to achieve sampling sufficiency for the overall floristic woody composition introduced in these sites. Original soil type and vegetation formation of revegetated sites were determined based on surrounding areas, satellite images, and soil profiles. Geographic coordinates were recorded on the center of each study site, with a Garmin GPS 40 device, datum SAD 69 (Table 1). 
Table 1. Some characteristics of study sites.

\begin{tabular}{|c|c|c|c|c|c|}
\hline Location & $\begin{array}{l}\text { Geographic } \\
\text { coordinates } \\
(\text { SAD 69) }\end{array}$ & $\begin{array}{l}\text { Revegetated } \\
\text { extension } \\
\text { (ha) }\end{array}$ & $\begin{array}{l}\text { Number of } \\
\text { planted } \\
\text { individuals }\end{array}$ & $\begin{array}{l}\text { Number of } \\
\text { species }\end{array}$ & $\begin{array}{l}\text { Number of } \\
\text { sampled } \\
\text { individuals }\end{array}$ \\
\hline 1 - SHIS QI 29 & $\begin{array}{l}15^{\circ} 48^{\prime} 44^{\prime \prime} \mathrm{S} \\
47^{\circ} 47^{\prime} 28^{\prime \prime} \mathrm{W}\end{array}$ & 5.4 & 1,514 & 32 & 400 \\
\hline 2 - Aeroporto JK & $\begin{array}{l}15^{\circ} 52^{\prime} 22^{\prime \prime} \mathrm{S} \\
47^{\circ} 53,11^{\prime \prime} \mathrm{W}\end{array}$ & 72.1 & 29,000 & 62 & 900 \\
\hline 3 - REFESA & $\begin{array}{l}15^{\circ} 47^{\prime} 14, \mathrm{~S} \\
47^{\circ} 57^{\prime} 07^{\prime \prime} \mathrm{W}\end{array}$ & 6.7 & 4,200 & 34 & 400 \\
\hline 4 - Parna de Bsb & $\begin{array}{l}15^{\circ} 44^{\prime} 06^{\prime \prime} \mathrm{S} \\
47^{\circ} 55^{\prime} 46^{\prime \prime} \mathrm{W}\end{array}$ & 1.1 & 690 & 32 & 400 \\
\hline 5 - Granja do Torto & $\begin{array}{l}15^{\circ} 42^{\prime} 43^{\prime \prime} \mathrm{S} \\
47^{\circ} 54^{\prime} 36^{\prime \prime} \mathrm{W}\end{array}$ & 16.4 & 5,410 & 21 & 400 \\
\hline $\begin{array}{l}6-\mathrm{BR} 060, \mathrm{Km} \\
14,5\end{array}$ & $\begin{array}{l}15^{\circ} 56^{\prime} 27^{\prime \prime} \mathrm{S} \\
48^{\circ} 09^{\prime} 31^{\prime \prime} \mathrm{W}\end{array}$ & 21.4 & 5,940 & 14 & 400 \\
\hline 7 - DF $130, \mathrm{Km} 8,5$ & $\begin{array}{l}15^{\circ} 45^{\prime} 26^{\prime \prime} \mathrm{S} \\
47^{\circ} 39^{\prime} 22^{\prime \prime} \mathrm{W}\end{array}$ & 4.5 & 2,530 & 20 & 400 \\
\hline 8- Arboreto UnB & $\begin{array}{l}15^{\circ} 44^{\prime} 20 ” \mathrm{~S} \\
47^{\circ} 53^{\prime} 02^{\prime \prime} \mathrm{W}\end{array}$ & 4.9 & 1,350 & 16 & 400 \\
\hline 9 - SMPW Q. 25 & $\begin{array}{l}15^{\circ} 54^{\prime} 01^{\prime}, \mathrm{S} \\
47^{\circ} 54^{\prime} 58^{\prime}, \mathrm{W}\end{array}$ & 6.3 & 6,000 & 19 & 400 \\
\hline 10 - DF 430 & $\begin{array}{l}15^{\circ} 40^{\prime} 01{ }^{\prime \prime} \mathrm{S} \\
48^{\circ} 10^{\prime} 47^{\prime \prime} \mathrm{W}\end{array}$ & 4.2 & 1,400 & 13 & 400 \\
\hline
\end{tabular}

\section{Data treatment}

Number of individuals of each species was relativized according to the sample number in each revegetated site in order to determine the abundance

$$
\begin{aligned}
& \text { Frequency - } F_{i}=\frac{\# \text { of sites } \text { with species } i}{\text { total } \# \text { of sites }} \times 100 \\
& \text { Abundance - } A b_{i}=\frac{\# \text { individuals of species } i}{\text { total number of individuals }} \times 100
\end{aligned}
$$

of each species within the floristic composition. The frequency and abundance of species were calculated according to Dajoz $(1983 ; 2005)$ :
Identified woody species were classified according to origin (autochthonous or allochthonous), habit (tree or shrub), natural habitat (savanna or forest), ecological group (pioneer, secondary, climax), and dispersal syndrome (anemochory, autocory, hydrochory, zoochory), based on Durigan and Silveira (1999), Duboc (2004), Duboc and Guerrini (2007), Carvallho (2008), Mendonça et al. (2008b) and Corrêa (2009). Regression analyses were performed among number of species, number of individuals, frequency of species, and abundance of species by using Matlab software, version 2009.

\section{RESULTS}

The sampling of 4,500 individuals along 143 hectares in ten surveyed sites was necessary to achieve stability tendency in the rarefaction curve (Figure 1). In this 143 hectares, which represents $4 \%$ of the extension degraded by mining in the Brazilian Federal District - BFD (CORRÊA et. al., 2004), there were 103 species of trees and 13 species of shrubs (Table 2). The survey of the first four sites assembled $92 \%$ of the species found in the study, while the survey of further six sites added nine species to the overall floristic composition.

Among the 116 species found, 92 (79\%) are autochthonous to Cerrado biome. Seedlings planted in sites were distributed in 36 botanical families (Table 2). Fabaceae was the family with the highest species richness (33 species), followed by Bignoniaceae (11), Malvaceae (11) and Anacardiaceae (7). The remaining families were represented by up to four species each one. Species of Anarcadiaceae, Bignoniaceae and Fabaceae were 
present in all the investigated sites, with a total of 51

species, or $44 \%$ of the floristic richness.

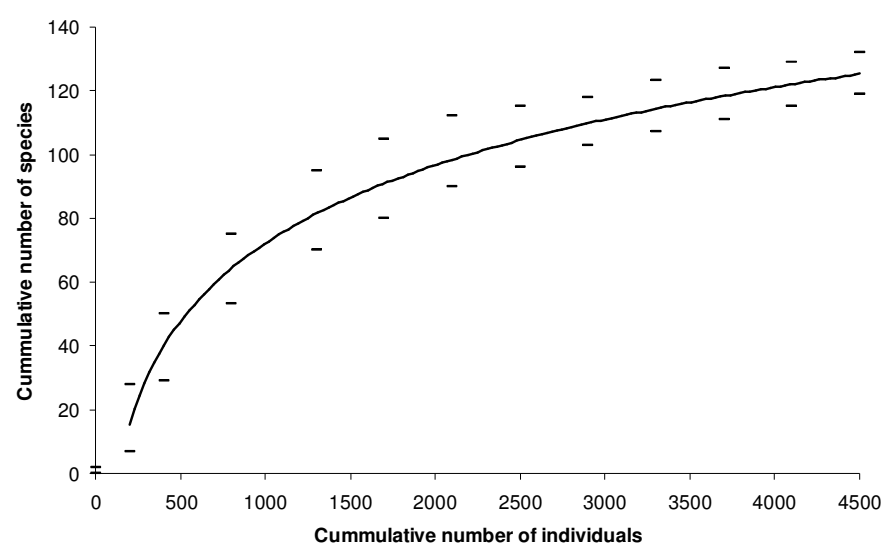

Figure 1. Species rarefaction curve for plants sampled in ten revegetated mining sites in the Brazilian Federal District - BFD.

Surveyed mining sites were revegetated with communities composed of 13 to 62 woody species (Table 1), with density of $467 \pm 222$ plants ha $^{-1}$ or a spacing of $4 \times 5 \mathrm{~m}$ among plants. Average number of species per revegetated site was of $26 \pm$ 15 , and only one project used more than 34 species (Table 1). The majority of projects $(60 \%)$ employed between 13 and 21 woody species. Twenty one allochthonous species to Cerrado were found in the sites, which corresponded to $18 \%$ of the floristic composition. It was not possible to determine the species of three identified genus (Table 2). Among the 92 autochthonous species found, 47 occur naturally in forest and savanna formations of Cerrado, 37 occur exclusively in forest formations and eight species are exclusive to savannic formations (Table 2). About a quarter (26\%) of the autochthonous species introduced in the sites are pioneer species, $37 \%$ are secondary and $3 \%$ are climax species. The remaining $34 \%$ of species have more than one ecological group classification (DURIGAN; SILVEIRA, 1999; DUBOC, 2004; DUBOC; GUERRINI, 2007; CARVALHO, 2008; MENDONÇA et al., 2008b; CORRÊA, 2009). More than half $(51 \%)$ of the autochthonous species identified in the sites present zoochorous dispersal syndrome, while $45 \%$ are anemochorous species, and $4 \%$ disperse by either autocory or hydrochory.

Of the 116 species found in the ten surveyed locations, 61 occurred in only one of the revegetated sites $(\mathrm{Fi}=10 \%), 21$ species were recorded in two sites $(\mathrm{Fi}=20 \%)$ and 15 species occurred in five or more sites $(\mathrm{Fi} \geq 50 \%)$. Only Myracrodruon urundeuva Allemão was found in all revegetated sites, while Anadenanthera macrocarpa (Benth.)
Brenan was present in $80 \%$ of the surveyed locations (Table 2).

Among the 4,500 individuals sampled in sites, $9.4 \%$ were $M$. urundeuva, followed in abundance by A. macrocarpa (5.6\%) and Triplaris brasiliana Cham. (4.8\%). The 16 most abundant species accounted for $55 \%$ of the number of sampled individuals. They were M. urundeuva (9.4\%), A. macrocarpa (5.6\%), T. brasiliana (4.8\%), Handroanthus albus (Cham.) Mattos (3.8\%), Dipteryx alata Vogel (3.4\%), Jacaranda macrantha Cham. (3.3\%), Genipa americana L. (3.1\%), Tabebuia roseoalba (Ridl.) Sandwith (3.1), Inga laurina (Sw.) Willd. (3.0), Myroxylon peruiferum L. f. (2.9\%), Guazuma ulmifolia Lam (2.4\%), Senegalia polyphylla (DC) Britton. Rose (2.2\%), Dalbergia miscolobium Benth. (2.1\%), Tabebuia aurea Benth. \& Hook. f. ex S. Moore (2.1\%), Inga cylindrica (Vell.) Mart. (2.0\%) and Plathymenia reticulata Benth. (2.0\%). Of these, J. macrantha is allochthonous to Cerrado biome. Among the 15 most abundant autochthonous species eight are exclusive of forest formations, while seven occur in savannas and forests. Ten of these species have anemochorous dispersal syndrome, four are zoochorous, and four species are pioneers. Eight of the 16 most abundant species are Fabaceae, while other four are Bignoniaceae. The other four species among the 16 most abundant ones belong to four distinct families (Table 2). The other $45 \%$ of the individuals found in the sites are distributed among the other 100 species of 30 different families. The families with the largest numbers of plants were Fabaceae - 1,622 or $36 \%$, Bignoniaceae - 719 or $16 \%$, and Anacardiaceae, with 541 individuals or $12 \%$ of sampled plants (Table 2). The frequency of 
species in sites influenced species abundance $\left(\mathrm{R}^{2}=\right.$ $0.66 ; \mathrm{P}<0.05)$.

Among the individuals sampled, $90.4 \%$ were of autochthonous trees, $1.7 \%$ were autochthonous shrubs and the remaining $7.9 \%$ were trees or shrubs allochthonous to Cerrado. The individuals of autochthonous species from forest formations totaled $46.1 \%$ of sampled plants, while those from savanna formations accounted for $4.6 \%$ of the same. Plants that occur naturally in forest and savanna formations amounted to $41.4 \%$ of the total sampled individuals. Autochthonous pioneer species represented $21.3 \%$ of the individuals sampled, and $21.7 \%$ were secondary species. Climax species accounted for $5 \%$, and $44.1 \%$ of the individuals had more than one classification for ecological group. Regarding dispersal syndrome, $53.5 \%$ of sampled individuals were of anemochorous species, $33.3 \%$ of zoochorous species, and $5.3 \%$ were individuals of either autochorous or hydrochorous species (DURIGAN; SILVEIRA, 1999; DUBOC, 2004; DUBOC; GUERRINI, 2007; CARVALHO, 2008; MENDONÇA et al., 2008b; CORRÊA, 2009).

Table 2. Taxon, frequency (Fi), abundance (Abi) and some characteristics of the woody species found in study sites.

\begin{tabular}{|c|c|c|c|c|c|c|}
\hline Taxon & $\begin{array}{l}\mathbf{F i} \\
(\%)\end{array}$ & $\begin{array}{l}\text { Abi } \\
(\%)\end{array}$ & Habit & Habitat & $\begin{array}{l}\text { Ecological } \\
\text { group }\end{array}$ & $\begin{array}{l}\text { Dispersal } \\
\text { syndrome }\end{array}$ \\
\hline \multicolumn{7}{|l|}{$\overline{\text { ANACARDIACEAE }}$} \\
\hline Anacardium occidentale L. & 10 & $<0.1$ & $\mathrm{~T}$ & $\mathrm{~S}, \mathrm{~F}$ & $\mathrm{p}, \mathrm{s}, \mathrm{c}$ & $\mathrm{z}$ \\
\hline $\begin{array}{l}\text { Astronium fraxinifolium } \\
\text { Schott ex Spreng }\end{array}$ & 20 & 0.7 & $\mathrm{~T}$ & $\mathrm{~S}, \mathrm{~F}$ & $\mathrm{p}, \mathrm{s}$ & a \\
\hline Astronium graveolens Jacq. & 20 & 0.8 & $\mathrm{~T}$ & $\mathrm{~F}$ & $\mathrm{~s}$ & a \\
\hline Lithraea molleoides Engl. & 10 & 0.3 & $\mathrm{~T}$ & $\mathrm{~F}$ & $\mathrm{p}$ & z \\
\hline $\begin{array}{l}\dagger \text { Myracrodruon urundeuva } \\
\text { Allemão }\end{array}$ & 100 & 9.4 & $\mathrm{~T}$ & $\mathrm{~F}$ & $\mathrm{~s}, \mathrm{c}$ & a \\
\hline Schinus terebinthifolius Raddi & 10 & 0.1 & $\mathrm{~T}$ & $\mathrm{~F}$ & $\mathrm{p}, \mathrm{s}$ & $\mathrm{z}$ \\
\hline $\begin{array}{l}\text { Tapirira guianensis Aubl. } \\
\text { ANNONACEAE }\end{array}$ & 50 & 0.9 & $\mathrm{~T}$ & $\mathrm{~S}, \mathrm{~F}$ & $\mathrm{p}, \mathrm{s}$ & $\mathrm{z}$ \\
\hline $\begin{array}{l}\text { Xylopia aromatica (Lam.) } \\
\text { Mart. } \\
\text { APOCYNACEAE }\end{array}$ & 20 & 0.4 & $\mathrm{~T}$ & $\mathrm{~S}$ & $\mathrm{p}$ & z \\
\hline $\begin{array}{l}\text { Aspidosperma macrocarpon } \\
\text { Mart. }\end{array}$ & 30 & 0.5 & $\mathrm{~T}$ & $\mathrm{~S}, \mathrm{~F}$ & S & $\mathrm{a}$ \\
\hline $\begin{array}{l}\text { Aspidosperma subincanum } \\
\text { Mart. }\end{array}$ & 10 & 0.1 & $\mathrm{~T}$ & $\mathrm{~F}$ & $\mathrm{~S}$ & $\mathrm{a}, \mathrm{z}$ \\
\hline$\dagger$ Hancornia speciosa Gomez & 30 & 0.4 & $\mathrm{~T}$ & $\mathrm{~S}, \mathrm{~F}$ & $\mathrm{~s}$ & $\mathrm{Z}$ \\
\hline $\begin{array}{l}\text { *Nerium oleander L. } \\
\text { ARALIACEAE }\end{array}$ & 10 & $<0.1$ & $\mathrm{Sb}$ & exotic & & \\
\hline $\begin{array}{l}\text { *Schefflera actinophylla } \\
\text { (Endl.) Harms } \\
\text { BIGNONIACEAE }\end{array}$ & 10 & $<0.1$ & $\mathrm{~T}$ & exotic & & $\mathrm{z}$ \\
\hline $\begin{array}{l}\text { Cybistax antisyphilitica } \\
\text { (Mart.) Mart. }\end{array}$ & 40 & 1.7 & $\mathrm{~T}$ & $\mathrm{~S}, \mathrm{~F}$ & $\mathrm{~s}$ & $\mathrm{a}$ \\
\hline Jacaranda macrantha Cham. & 40 & 3.3 & $\mathrm{~T}$ & exotic & $\mathrm{p}$ & $\mathrm{a}$ \\
\hline $\begin{array}{l}\text { Handroanthus albus (Cham.) } \\
\text { Mattos }\end{array}$ & 70 & 3.8 & $\mathrm{~T}$ & $\mathrm{~S}, \mathrm{~F}$ & $\mathrm{c}$ & $\mathrm{a}$ \\
\hline $\begin{array}{l}\text { Handroanthus chrysotrichus } \\
\text { (Mart.ex DC) Mattos }\end{array}$ & 30 & 0.4 & $\mathrm{~T}$ & $\mathrm{~F}$ & s & $\mathrm{a}$ \\
\hline $\begin{array}{l}\dagger \text { Tabebuia aurea Benth. \& } \\
\text { Hook. f. ex S. Moore }\end{array}$ & 70 & 2.1 & $\mathrm{~T}$ & $\mathrm{~S}, \mathrm{~F}$ & $\mathrm{~s}$ & $\mathrm{a}$ \\
\hline $\begin{array}{l}\text { Tabebuia impetiginosa (Mart. } \\
\text { ex DC.) Standl. }\end{array}$ & 30 & 0.7 & $\mathrm{~T}$ & $\mathrm{~F}$ & $\mathrm{~s}$ & $\mathrm{a}$ \\
\hline
\end{tabular}




\begin{tabular}{|c|c|c|c|c|c|c|}
\hline $\begin{array}{l}\text { Tabebuia ochracea (Cham.) } \\
\text { Standl. }\end{array}$ & 10 & $<0.1$ & $\mathrm{~T}$ & $S, F$ & s & $\mathrm{a}$ \\
\hline Tabebuia roseoalba (Ridl.) & 70 & 3.1 & $\mathrm{~T}$ & $\mathrm{~F}$ & $\mathrm{~s}$ & $\mathrm{a}$ \\
\hline Sandwith & & & & & & \\
\hline *Tabebuia sp. & 10 & 0.8 & $\mathrm{Sb}$ & exotic & & \\
\hline *† Stenolobium stans (L.) & 20 & 0.2 & $\mathrm{Sb}$ & exotic & & \\
\hline Seem & & & & & & \\
\hline $\begin{array}{l}\text { Zeyheria montana Mart. } \\
\text { BORAGINACEAE }\end{array}$ & 10 & $<0.1$ & $\mathrm{Sb}$ & $\mathrm{S}, \mathrm{F}$ & $\mathrm{p}$ & a \\
\hline $\begin{array}{l}\text { Cordia sellowiana Cham. } \\
\text { CALOPHYLLACEAE }\end{array}$ & 10 & 0.3 & $\mathrm{~T}$ & $\mathrm{~F}$ & $\mathrm{p}$ & $\mathrm{a}$ \\
\hline $\begin{array}{l}\text { Calophyllum brasiliense } \\
\text { Cambess. }\end{array}$ & 30 & 1.1 & $\mathrm{~T}$ & $\mathrm{~F}$ & $\mathrm{~s}, \mathrm{c}$ & $\mathrm{h}, \mathrm{z}$ \\
\hline $\begin{array}{l}\dagger \text { \#Kielmeyera coriacea } \text { Mart. } \\
\text { \& Zucc. } \\
\text { CARYOCARACEAE }\end{array}$ & 20 & $<0.1$ & $\mathrm{~T}$ & $\mathrm{~S}, \mathrm{~F}$ & $\mathrm{p}$ & $\mathrm{a}$ \\
\hline $\begin{array}{l}\dagger \# \text { Caryocar brasiliense } \\
\text { Cambess. } \\
\text { CELASTRACEAE }\end{array}$ & 30 & 0.3 & $\mathrm{~T}$ & $\mathrm{~S}, \mathrm{~F}$ & $\mathrm{p}, \mathrm{s}, \mathrm{c}$ & $\mathrm{z}$ \\
\hline $\begin{array}{l}\dagger \text { Plenckia populnea } \text { Reissek } \\
\text { CHRYSOBALANACEAE }\end{array}$ & 10 & $<0.1$ & $\mathrm{~T}$ & $\mathrm{~S}, \mathrm{~F}$ & $\mathrm{p}$ & a \\
\hline $\begin{array}{l}\text { Couepia grandiflora (Mart. \& } \\
\text { Zucc.) Benth. ex Hook. f. } \\
\text { CLUSIACEAE }\end{array}$ & 10 & 0.1 & $\mathrm{~T}$ & $\mathrm{~S}, \mathrm{~F}$, & $\mathrm{s}$ & $\mathrm{z}$ \\
\hline $\begin{array}{l}\text { Clusia } \text { sp. } \\
\text { COMBRETACEAE }\end{array}$ & 20 & 0.1 & $\mathrm{~T}$ & & & \\
\hline Terminalia argentea Mart. & $\begin{array}{l}30 \\
10\end{array}$ & $\begin{array}{l}1.2 \\
0.3\end{array}$ & $\begin{array}{l}\mathrm{T} \\
\mathrm{T}\end{array}$ & $\begin{array}{l}S, F \\
S, F\end{array}$ & $\begin{array}{l}\mathrm{p} \\
\mathrm{s}\end{array}$ & $\begin{array}{l}\mathrm{a}, \mathrm{h} \\
\mathrm{a}\end{array}$ \\
\hline $\begin{array}{l}\text { Terminalia fagifolia Mart. } \\
\text { Terminalia phaeocarpa } \\
\text { Eichler } \\
\text { DILLENIACEAE }\end{array}$ & 10 & $<0.1$ & $\mathrm{~T}$ & $\mathrm{~F}$ & $\mathrm{p}$ & $\mathrm{a}$ \\
\hline $\begin{array}{l}\text { Curatella americana } \mathrm{L} . \\
\text { ERYTHROXYLACEAE }\end{array}$ & 10 & $<0.1$ & $\mathrm{~T}$ & $\mathrm{~S}, \mathrm{~F}$ & $\mathrm{c}$ & $\mathrm{z}$ \\
\hline $\begin{array}{l}\text { \# Erythroxylum suberosum A. } \\
\text { St.-Hil. } \\
\text { EUPHORBIACEAE }\end{array}$ & 10 & $<0.1$ & $\mathrm{Sb}$ & S & $\mathrm{s}$ & $\mathrm{z}$ \\
\hline $\begin{array}{l}\text { Margaritaria nobilis L.f. } \\
* \dagger \text { Ricinus communis L. }\end{array}$ & $\begin{array}{l}10 \\
10\end{array}$ & $\begin{array}{l}<0.1 \\
<0.1\end{array}$ & $\begin{array}{l}\mathrm{T} \\
\mathrm{Sb}\end{array}$ & $\begin{array}{l}\text { F } \\
\text { exotic }\end{array}$ & $\mathrm{s}$ & $\mathrm{t}, \mathrm{z}$ \\
\hline $\begin{array}{l}\text { Sapium glandulatum (Vell.) } \\
\text { Pax } \\
\text { FABACEAE }\end{array}$ & 10 & $<0.1$ & $\mathrm{Sb}$ & $\mathrm{S}, \mathrm{F}$ & $\mathrm{p}$ & $\mathrm{z}$ \\
\hline $\begin{array}{l}\text { Amburana cearensis } \\
\text { (Allemão) A.C. Sm. }\end{array}$ & 10 & 0.2 & $\mathrm{~T}$ & $\mathrm{~F}$ & $\mathrm{~s}$ & $\mathrm{a}$ \\
\hline $\begin{array}{l}\text { † Anadenanthera macrocarpa } \\
\text { (Benth.) Brenan }\end{array}$ & 80 & 5.6 & $\mathrm{~T}$ & $\mathrm{~F}$ & $\mathrm{~s}, \mathrm{c}$ & a \\
\hline$\dagger$ Bauhinia sp. & 10 & 0.9 & $\mathrm{~T}$ & & & \\
\hline *Caesalpinia echinata Lam. & 10 & 0.1 & $\mathrm{~T}$ & exotic & & \\
\hline *Calliandra sp. & 10 & 0.2 & $\mathrm{Sb}$ & exotic & & \\
\hline Copaifera langsdorffii Desf. & 60 & 1.8 & $\mathrm{~T}$ & $\mathrm{~F}$ & $\mathrm{~s}, \mathrm{c}$ & $\mathrm{z}$ \\
\hline $\begin{array}{l}\dagger \text { \#Dalbergia miscolobium } \\
\text { Benth. }\end{array}$ & 60 & 2.1 & $\mathrm{~T}$ & $\mathrm{~S}, \mathrm{~F}$ & $\mathrm{p}$ & $\mathrm{a}$ \\
\hline$\dagger$ Dimorphandra mollis Benth. & 10 & $<0.1$ & $\mathrm{~T}$ & S & $\mathrm{p}$ & $\mathrm{z}$ \\
\hline Dipteryx alata Vogel & 50 & 3.4 & $\mathrm{~T}$ & $\mathrm{~S}, \mathrm{~F}$ & $\mathrm{~s}, \mathrm{c}$ & $\mathrm{t}, \mathrm{z}$ \\
\hline
\end{tabular}




\begin{tabular}{|c|c|c|c|c|c|c|}
\hline $\begin{array}{l}\text { Enterolobium } \\
\text { contortisiliquum (Vell.) } \\
\text { Morong }\end{array}$ & 20 & 0.5 & $\mathrm{~T}$ & $\mathrm{~F}$ & $\mathrm{p}, \mathrm{s}$ & $\mathrm{t}, \mathrm{z}$ \\
\hline $\begin{array}{l}\text { Enterolobium gummiferum } \\
\text { Benth. }\end{array}$ & 10 & $<0.1$ & $\mathrm{~T}$ & $\mathrm{~S}, \mathrm{~F}$ & $\mathrm{p}, \mathrm{s}$ & $\mathrm{t}, \mathrm{z}$ \\
\hline Erythrina verna Vell. & 20 & $<0.1$ & $\mathrm{~T}$ & $\mathrm{~S}, \mathrm{~F}$ & s & $\mathrm{a}, \mathrm{z}$ \\
\hline $\begin{array}{l}\text { Hymenaea courbaril var. } \\
\text { stilbocarpa (Hayne) Y.T. Lee } \\
\text { \& Langenh. }\end{array}$ & 60 & 1.9 & $\mathrm{~T}$ & $\mathrm{~F}$ & $\mathrm{~s}, \mathrm{c}$ & $\mathrm{t}, \mathrm{z}$ \\
\hline $\begin{array}{l}\text { †Hymenaea stigonocarpa } \\
\text { Mart. ex. Hayne }\end{array}$ & 70 & 1.2 & $\mathrm{~T}$ & $\mathrm{~S}, \mathrm{~F}$ & $\mathrm{~s}, \mathrm{c}$ & $\mathrm{t}, \mathrm{z}$ \\
\hline Inga cylindrica (Vell.) Mart. & 30 & 2.0 & $\mathrm{~T}$ & $\mathrm{~F}$ & $\mathrm{p}$ & $\mathrm{h}, \mathrm{z}$ \\
\hline Inga edulis var. edulis & 20 & 0.5 & $\mathrm{~T}$ & $\mathrm{~F}$ & S & h, $\mathrm{z}$ \\
\hline Inga laurina (Sw.) Willd. & 70 & 3.0 & $\mathrm{~T}$ & $\mathrm{~F}$ & $\mathrm{~s}$ & h, $\mathrm{z}$ \\
\hline $\begin{array}{l}* \dagger \text { Leucaena leucocephala } \\
\text { (Lam.) de Wit. }\end{array}$ & 10 & $<0.1$ & $\mathrm{~T}$ & exotic & & \\
\hline *Libidibia ferrea Mart. & 30 & 1.5 & $\mathrm{~T}$ & exotic & & \\
\hline$\dagger$ Machaerium opacum Vogel & 10 & $<0.1$ & $\mathrm{~T}$ & $\mathrm{~S}, \mathrm{~F}$ & $\mathrm{p}, \mathrm{s}$ & $\mathrm{a}$ \\
\hline † Mimosa claussenii Benth. & 10 & $<0.1$ & $\mathrm{Sb}$ & $\mathrm{S}$ & $\mathrm{p}$ & $\mathrm{t}$ \\
\hline Mimosa sp. & 10 & $<0.1$ & $\mathrm{Sb}$ & & & \\
\hline Myroxylon peruiferum L. f. & 50 & 2.9 & $\mathrm{~T}$ & $\mathrm{~F}$ & $\mathrm{~s}, \mathrm{c}$ & $\mathrm{a}$ \\
\hline Ormosia stipularis Ducke & 10 & 0.3 & $\mathrm{~T}$ & $\mathrm{~F}$ & $\mathrm{~s}, \mathrm{c}$ & $\mathrm{t}, \mathrm{z}$ \\
\hline $\begin{array}{l}\text { Piptadenia gonoacantha } \\
\text { (Mart.) Macbr. }\end{array}$ & 10 & 1.5 & $\mathrm{~T}$ & $\mathrm{~F}$ & $\mathrm{p}$ & $\mathrm{t}$ \\
\hline $\begin{array}{l}+ \text { Plathymenia reticulata } \\
\text { Benth. }\end{array}$ & 20 & 2.0 & $\mathrm{~T}$ & $\mathrm{~S}, \mathrm{~F}$ & $\mathrm{p}, \mathrm{s}, \mathrm{c}$ & $\mathrm{a}$ \\
\hline Platypodium elegans Vogel & 10 & 0.6 & $\mathrm{~T}$ & $\mathrm{~F}$ & $\mathrm{~s}$ & $\mathrm{a}$ \\
\hline $\begin{array}{l}\dagger \text { Pterodon pubescens (Benth.) } \\
\text { Benth. }\end{array}$ & 10 & $<0.1$ & $\mathrm{~T}$ & $\mathrm{~S}, \mathrm{~F}$ & $\mathrm{~s}, \mathrm{c}$ & $\mathrm{a}$ \\
\hline Pterogyne nitens Tul. & 10 & 0.4 & $\mathrm{~T}$ & exotic & & \\
\hline $\begin{array}{l}\text { \#Sclerolobium paniculatum } \\
\text { Vogel }\end{array}$ & 10 & $<0.1$ & $\mathrm{~T}$ & $\mathrm{~S}, \mathrm{~F}$ & $\mathrm{~s}$ & $\mathrm{a}, \mathrm{z}$ \\
\hline $\begin{array}{l}\text { Senegalia polyphylla (DC) } \\
\text { Britton. Rose }\end{array}$ & 40 & 2.2 & $\mathrm{~T}$ & $\mathrm{~S}, \mathrm{~F}$ & $\mathrm{p}$ & $\mathrm{t}$ \\
\hline $\begin{array}{l}\text { † \#Stryphnodendron } \\
\text { adstringens (Mart.) Coville }\end{array}$ & 20 & 0.4 & $\mathrm{~T}$ & $\mathrm{~S}$ & $\mathrm{p}, \mathrm{s}$ & $\mathrm{t}$ \\
\hline $\begin{array}{l}\text { *Tamarindus indica L. } \\
\text { ICACINACEAE }\end{array}$ & 10 & 0.1 & $\mathrm{~T}$ & exotic & & \\
\hline $\begin{array}{l}\text { Emmotum nitens (Benth.) } \\
\text { Miers } \\
\text { LAMIACEAE }\end{array}$ & 10 & 0.1 & $\mathrm{~T}$ & $\mathrm{~S}, \mathrm{~F}$ & $\mathrm{~s}$ & $\mathrm{Z}$ \\
\hline $\begin{array}{l}\text { Aegiphila lhotskiana Cham. } \\
\text { LECYTIDACEAE }\end{array}$ & 10 & 1.3 & $\mathrm{~T}$ & $\mathrm{~S}$ & $\mathrm{p}$ & $\mathrm{Z}$ \\
\hline $\begin{array}{l}\text { Cariniana estrellensis } \\
\text { (Raddi) Kuntze } \\
\text { LYTHRACEAE }\end{array}$ & 30 & 1.0 & $\mathrm{~T}$ & $\mathrm{~F}$ & $\mathrm{~s}, \mathrm{c}$ & $\mathrm{a}$ \\
\hline $\begin{array}{l}\text { Physocalymma scaberrimum } \\
\text { Pohl } \\
\text { MALVACEAE }\end{array}$ & 10 & 0.6 & $\mathrm{~T}$ & $\mathrm{~S}, \mathrm{~F}$ & $\mathrm{p}$ & $\mathrm{a}$ \\
\hline Apeiba tibourbou Aubl. & 10 & 0.1 & $\mathrm{~T}$ & $\mathrm{~F}$ & $\mathrm{p}, \mathrm{s}$ & $\mathrm{z}$ \\
\hline Chorisia speciosa A. St.-Hil. & 20 & 0.9 & $\mathrm{~T}$ & $\mathrm{~F}$ & $\mathrm{~S}$ & $\mathrm{a}$ \\
\hline $\begin{array}{l}\text { Eriotheca gracilipes (K. } \\
\text { Schum.) A. Robyns }\end{array}$ & 20 & 0.3 & $\mathrm{~T}$ & $\mathrm{~S}, \mathrm{~F}$ & $\mathrm{~s}$ & $\mathrm{a}$ \\
\hline
\end{tabular}


$\dagger$ Eriotheca pubescens (Mart.

10

$0.7 \quad \mathrm{~T}$

S, F

a

\& Zucc.) Schott \& Endl.

$\uparrow$ Guazuma ulmifolia Lam

†Luehea divaricata Mart.

30

30

Luehea grandiflora Mart.

*Ochroma pyramidale (Cav.

ex Lam.) Urb.

Pseudobombax longiflorum

(Martius \& Zuccarini) A.

Robyns

Pseudobombax tomentosum

(C. Martius \& Zuccarini)

Robyns

Sterculia striata A. St.-Hil. \& 20

Naudin

MELASTOMATACEAE

$\dagger$ Tibouchina stenocarpa

(DC.) Cogn.

MELIACEAE

Cedrela fissilis Vell.

* Melia azedarach $\mathrm{L}$.

* Swietenia macrophylla King

MORACEAE

*Ficus benjamina L.

*Morus nigra L.

MYRTACEAE

Blepharocalyx salicifolius

(Kunth) O. Berg

$\dagger$ Eugenia dysenterica DC.

*Eugenia uniflora L.

Psidium guianense Sw.

$\dagger$ Psidium myrsinoides $\mathrm{O}$.

Berg

NYCTAGINACEAE

$\dagger$ \#Guapira noxia (Netto)

Lundell

OCHNACEAE

Ouratea castaneifolia (DC.)

Engl.

† \#Ouratea hexasperma (A.

St.-Hil.) Baill.

POLYGONACEAE

Triplaris brasiliana Cham. 50

PROTEACEAE

40

$0.6 \mathrm{~T}$

S, F

S, F

$\mathrm{F}$

$\mathrm{F}$

exotic

20

20

$1.1 \quad \mathrm{~T}$

S, F

\section{$\mathrm{p}$}

a, z

$1.8 \mathrm{~T}$

$<0.1 \quad \mathrm{~T}$

$\mathrm{p}, \mathrm{s}$

a

$\mathrm{a}$

$0.2 \mathrm{~T}$

S, F

S

a

$1.0 \mathrm{~T} \quad \mathrm{~F}$

S, C

Z

a

F

0.6

$\begin{array}{lll}20 & 1.6 \quad \mathrm{~T}\end{array}$

$20 \quad 1.0 \quad \mathrm{~T}$

10

$0.3 \mathrm{~T}$

F

exotic

exotic

$\begin{array}{llll}10 & 0.2 & \mathrm{~T} & \text { exotic }\end{array}$

$\begin{array}{llll}10 & 0.2 & \mathrm{~T} & \text { exotic }\end{array}$

40

$0.7 \quad \mathrm{~T}$

S, F

$\mathrm{p}, \mathrm{s}$

a

$\begin{array}{lll}30 & 0.9 & \mathrm{~T}\end{array}$

S, F

exotic

$\begin{array}{lll}10 & 0.2 & \mathrm{~T}\end{array}$

$\begin{array}{lll}10 & 0.2 & \mathrm{~T}\end{array}$

10

$0.8 \mathrm{Sb}$

$\mathrm{F}$

S

S, c

a

$\mathrm{s}, \mathrm{c}$

$10<0.1 \quad \mathrm{~T}$

S, F

Z

10

$<0.1 \quad \mathrm{~T}$

S, F

S, F

Z

*Grevillea robusta A. Cunn. 10

ex R. Br.

$\dagger$ \#Roupala montana Aubl. 10

RHAMNACEAE

*Hovenia dulcis Thunb.

Rhamnidium elaeocarpum

Reissek

$10<0.1 \quad \mathrm{~T}$

50

$4.8 \quad \mathrm{~T}$

F

a

exotic

$10<0.1 \quad \mathrm{~T}$

S, F

$\mathrm{p}, \mathrm{s}$

$\mathrm{t}, \mathrm{a}$

$\begin{array}{lll}10 & 0.1 & \mathrm{~T}\end{array}$

exotic

S, F

S

20

$0.1 \quad \mathrm{Sb}$

exotic

*Cydonia oblonga Mill.

$\begin{array}{llll}10 & 0.6 & \mathrm{~T} & \mathrm{~S}, \mathrm{~F} \\ & & & \\ 20 & 0.1 & \mathrm{Sb} & \text { exotic }\end{array}$

RUBIACEAE 


\begin{tabular}{|c|c|c|c|c|c|c|}
\hline $\begin{array}{l}\text { Alibertia macrophylla } \mathrm{K} . \\
\text { Schum. }\end{array}$ & 10 & 0.5 & $\mathrm{~T}$ & $\mathrm{~S}, \mathrm{~F}$ & $\mathrm{~s}$ & $\mathrm{z}$ \\
\hline Amaioua guianensis Aubl. & 10 & 0.1 & $\mathrm{~T}$ & $\mathrm{~F}$ & $\mathrm{p}, \mathrm{s}$ & $\mathrm{z}$ \\
\hline $\begin{array}{l}\text { Genipa americana L. } \\
\text { RUTACEAE }\end{array}$ & 60 & 3.1 & $\mathrm{~T}$ & $\mathrm{~F}$ & $\mathrm{~s}, \mathrm{c}$ & h, $\mathrm{z}$ \\
\hline $\begin{array}{l}\text { Zanthoxylum rhoifolium Lam. } \\
\text { SAPINDACEAE }\end{array}$ & 10 & 1.1 & $\mathrm{~T}$ & $\mathrm{~F}$ & $\mathrm{p}, \mathrm{s}, \mathrm{c}$ & $\mathrm{z}$ \\
\hline $\begin{array}{l}\text { Dilodendron bipinnatum } \\
\text { Radlk. }\end{array}$ & 10 & $<0.1$ & $\mathrm{~T}$ & $\mathrm{~F}$ & $\mathrm{p}$ & $\mathrm{z}$ \\
\hline $\begin{array}{l}\text { Magonia pubescens A. St.- } \\
\text { Hil. }\end{array}$ & 20 & 0.6 & $\mathrm{~T}$ & $\mathrm{~S}, \mathrm{~F}$ & s & $\mathrm{a}$ \\
\hline $\begin{array}{l}\text { Sapindus saponaria L. } \\
\text { SOLANACEAE }\end{array}$ & 20 & $<0.1$ & $\mathrm{~T}$ & $\mathrm{~F}$ & $\mathrm{p}, \mathrm{s}$ & $\mathrm{t}, \mathrm{z}$ \\
\hline $\begin{array}{l}\text { † Solanum lycocarpum A. St.- } \\
\text { Hil. } \\
\text { URTICACEAE }\end{array}$ & 10 & 0.4 & $\mathrm{Sb}$ & $\mathrm{S}, \mathrm{F}$ & $\mathrm{p}$ & $\mathrm{t}, \mathrm{z}$ \\
\hline $\begin{array}{l}\text { †Cecropia pachystachya } \\
\text { Trécul }\end{array}$ & 30 & 0.3 & $\mathrm{~T}$ & $\mathrm{~S}, \mathrm{~F}$ & $\mathrm{p}$ & $\mathrm{z}$ \\
\hline
\end{tabular}

*allochthonous (exotic) species to Cerrado biome; $\uparrow$ Species capable of spontaneously establishing in mine sites in Cerrado (CORRÊA et al., 2007); Habit: T (tree), Sb (schrub). Habitat: S (savanna), F (forest). Ecological group: p (pioneer), s (secondary), c (clímax). Dispersal syndrome: a (anemochory), t (autocory), h (hydrochory), z (zoochory); Sources: Durigan and Silveira (1999), Duboc (2004), Duboc and Guerrini (2007), Carvallho (2008), Mendonça et al. (2008b), Corrêa (2009).

\section{DISCUSSION}

The floristic survey in the revegetated mining sites confirmed the pattern of conspicuous use of autochthonous tree species, which were grouped into assemblies of low (13 species) and medium (62 species) species richness (BARBOSA et al., 2003; 2012). The average number of woody species planted in sites was of $26 \pm 15$, which is less than half the species richness naturally present in Cerrado's savannic formations - 65 to 162 woody species ha ${ }^{-1}$ (EITEN, 1994). A small proportion of individuals of allochthonous species were found in the revegetated sites $(7.9 \%)$, which suggests the accidental use (DAJOZ, 1983; 2005) of 20 out the 21 allochthonous species identified. Only $J$. macrantha, an allochthonous species to the Cerrado, presented a consistent pattern of intentional use, as it appeared in $40 \%$ of the surveyed locations and was the sixth most abundant species among the 116 found in the study sites (Table 2).

The preferential use of native species complies with successional models for ecological restoration (ENGEL; PARROTTA, 2003; RODRIGUES et al., 2009; MARTINS et al., 2012). However, high diversity of plant species in initial communities of successional models is reported as the most ecologically efficient approach for ecosystem restoration, because an elevated species richness (80 - 90 species) may include species of various ecological groups, architectures and dispersal syndromes (BARBOSA et al. 2003; 2012;
RODRIGUES et al., 2009). High diversity communities also provide high structural diversity, perches and food for animals, maximize interaction networks, ecological functions and ensure the growth and maintenance of ecological processes in revegetated sites (BARBOSA et al., 2003; 2012; ENGEL; PARROTTA, 2003; REIS et al., 2003; 2010; RODRIGUES et al., 2009). Among the surveyed sites, only one presented a number of species deemed close to ideal $(80-90)$ in its initial plant community (BARBOSA et al. 2003) (Table 1).

Current successional restoration models are based on spontaneous changes in plant communities established by man (ENGEL; PARROTTA, 2003). Species diversity and planting density are factors that strongly influence ecological succession in recovering ecosystems that start off from planted communities (BARBOSA et al., 2003; 2102; LYONS et al., 2005; MOKANY et al., 2008). The reduced species richness in the study sites was accompanied by a low planting density $(467 \pm 222$ plants $\mathrm{ha}^{-1}$ ), which falls short to the lower limit of woody plant density in savannic formations of Cerrado (660 - 1,990 woody plants ha ${ }^{-1}$ ) (EITEN, 1994; 2001). Based on species richness and plant density, the initial plant communities established in the mining sites are most probably ineffective for ecosystem restoration (BARBOSA et al., 2003; MOKANY et al., 2008; BARBOSA et al., 2012).

Biodiversity is an all-encompassing term and from the standpoint of ecosystem functioning, functional diversity rather than taxonomic diversity 
is the most relevant component of biodiversity (NAEEM, 2006). Ecological groups and dispersal syndromes of plant species are essential functional traits in ecological restoration projects (ENGEL; PAROTTA, 2003; KAGEYAMA et al., 2003; REIS et. al., 2003; BARBOSA; PIZO, 2006; ARAÚJO; REIS, 2009). Cerrado's autochthonous species from all ecological groups, dispersal syndromes, forest and savanna formations were found in the revegetated sites (Table 2). In this sense, the use of plant species with different traits and functions dominates the current restoration model applied to the mine sites in the Brazilian Federal District BFD.

But the presence of species with varied functional traits alone may not guarantee the success of a restoration project (BARBOSA et al., 2003), because species evenness must portray the functional diversity of a plant assembly (MOKANY et al., 2008). Half (51\%) of the 92 autochthonous species identified in the studied sites is dispersed by fauna but only $33.3 \%$ of sampled individuals were of zoochorous species, while $53.5 \%$ of individuals presented anemochorous dispersal syndrome. Thus, the number of plant species dispersed by animals in the revegetated sites does not reflect the intensity of interactions between fauna and flora due to the relatively low number of zoochorous individuals in the initial plant communities. Respect to the number of individuals, there was a fair proportion among plants of species from different ecological groups: the number of plants of pioneer and secondary species was similar, and $44.1 \%$ of sampled plants had more than one classification for ecological group (Table 2).

The mass ratio hypothesis argues that ecosystem processes in communities are largely shaped by the functional traits of dominant species (MOKANY et al., 2008). Abundance exerts a strong influence on species dominance (DAJOZ, 1983; 2005) and the 16 most abundant species in the sites, which were generally the most frequent species in the surveyed locations, amounted to $55 \%$ of the number of sampled individuals (Table 2). These species, which are likely to be shaping the evolution of the initial communities introduced in the sites, are trees, being 15 of them naturally occurring in forest formations of Cerrado and one species is allochthonous to the Cerrado biome - J. macrantha. Fabaceae and Bignoniaceae accounted for $52 \%$ of all sampled individuals (Table 2) and 11 species that were planted in more than half of the surveyed sites (Fi $\geq 50 \%)$ are among the 16 most abundant species. Based on values of frequency and abundance, there are 19 autochthonous species to the Cerrado biome and one allochthonous to it that have been systematically used in restoration projects of mine sites in the BFD.

Some studies claim that ecosystem functioning precludes the need for complete taxonomic diversity and can be maintained with a reduced number of species that present a large number of interspecific interactions (NAEEM, 2006; GUIMARÃES JR., 2009). Under such view, the use of low number of plant species for ecological restoration (Table 1) would not be a problem as long as the assembled plants provide a dense ecological web. However, tropical ecosystems are highly diverse to properly function with a small number of species (ANAND; DESROCHERS, 2004) and most species exists at low abundance levels in natural communities, like in Cerrado formations (EITEN, 1994; 2001; DAJOZ, 2005; LYONS et al., 2005). As such, the contribution of rare, keystone and less common species cannot be neglected in restoration projects because they aggregate as a whole measurable benefits to ecosystem functioning and diversity (LYONS et al., 2005). Rare and low abundance species also create ecosystem own identity, discriminate a community from other communities, and increase the various levels of diversity as a result (DAJOZ, 2005).

High diversity plant communities established on degraded sites has shown to be an effective concept for ecosystem restoration (BARBOSA et al., 2003; 2012; RODRIGUES et al., 2009). But researches have proposed the replacement of restoration concepts based on high diversity of species (BARBOSA et al., 2003; 2012) by the selection of a reduced number of species that could stimulate and accelerate ecological succession (REIS et al., 2003; 2010). Among the 92 autochthonous species identified (Table 2), Inga spp., Cecropia pachystachya Trécul, Solanum lycocarpum A. St.-Hil., and Genipa americana L. have been reported to stimulate succession due to their vegetative characteristics and the large number of animal species that use them (REIS et al. 2003; 2010; CORRÊA, 2009).

Generalist species for habitat, ecological group and dispersal/pollination syndrome exhibit interaction networks wider than specialist species (GUIMARÃES JR., 2009) and may be more adequate for restoration projects from the practical point of view. More than half of autochthonous species planted in the surveyed sites occur naturally in forest and savanna formations of Cerrado, 34\% had more than one classification for ecological group, $51 \%$ interact with fauna to disperse and $1 / 5$ of them are dispersed by more than one agent type 
(Table 2). These generalist traits can facilitate the restoration of interspecific relationships in recovering ecosystems (GUIMARÃES JR., 2009), although richer and denser plant assemblies are necessary in the studied sites (BARBOSA et al., 2003; 2012; LYONS et al., 2005; RODRIGUES et al., 2009). The 92 autochthonous woody species currently in use in restoration projects (Table 2) can compose functionally better plant communities than those found in the revegetated sites.

The studied scenario has shown that more than $90 \%$ of the individuals sampled in the sites were arboreal, being half of them of species exclusively found in forest formations (Table 2). The plant communities studied showed only six autochthonous shrub species accounting for only $1.7 \%$ of the sampled individuals. Thus, the initial communities established in the sites may be driving succession to pathways that unlikely will end up in similar savanna ecosystems (ENGEL; PAROTTA, 2003) as intended for restoration projects (Brazilian Federal Law Nr. 9.985/2000). Tree plantation based on the use of autochthonous species is still the main model for ecological restoration in Brazil (MARTINS et al., 2012) and so it is in the BFD (CORRÊA, 2009). But tree species represent a part of the organisms in tropical ecosystems, including forests (ENGEL; PAROTTA, 2003; KAGEYAMA et al., 2003). Tree establishment in areas of grasslands and savannas is considered a common mistake of restoration projects in Cerrado (ENGEL; PAROTTA, 2003). A considerable portion of the current knowledge on forest restoration does not apply to grassland and savanna formations, which makes the restoration of these ecosystems even more challenging (DURIGAN, 2003; DURIGAN; ENGEL, 2012).

\section{CONCLUSIONS}

The initial plant communities established in the study sites resemble the standards recommended by successional restoration models, but the floristic diversity and plant density fall below the values considered ideal.

There were 92 autochthonous species in the study sites, but only 19 of them were systematically used in the revegetation works. Among the 92 species there are representatives of various habitats, ecological groups and dispersal syndromes, and this set can be rearranged into initial communities denser and functionally more diverse than those established in surveyed locations.

Plant communities were composed almost exclusively of arboreal species, and they will probably lead succession towards the formation of forest ecosystems where previously inhabited savanna formations.

Plant communities found in each site will hardly drive succession towards the original ecosystems, chiefly due to the adopted floristic composition, low species diversity and reduced planting density.

\section{ACKNOWLEGMENTS}

Authors thank land owners who allowed surveying the study sites and reviewers whose comments improved the manuscript.

RESUMO: A extensão minerada no Distrito Federal - DF é proporcionalmente cinco vezes superior à média nacional. A restauração das comunidades vegetais nesses locais é necessária e obrigatória, e o plantio de espécies lenhosas é prática comum de restauração. Dessa forma, este trabalho visou identificar e avaliar a composição florística lenhosa introduzida em jazidas mineradas no DF como meio de restaurar o ecossistema natural. O levantamento florístico nas áreas selecionadas foi realizado por meio da amostragem de grupos de cem indivíduos, até se obter suficiência amostral. Foram amostradas 4.500 plantas em dez jazidas, que abrigavam 92 espécies lenhosas autóctones e 21 alóctones. As comunidades vegetais levantadas eram formadas por 13 a 62 espécies, plantadas a uma baixa densidade $-467 \pm 222$ plantas ha $^{-1}$. O uso preferencial de espécies autóctones nos projetos de revegetação os assemelham aos padrões recomendados pelos modelos sucessionais de restauração, mas a diversidade florística e densidade de plantas nas comunidades iniciais estão abaixo dos valores considerados ideais. Entre as 92 espécies identificadas há representantes de variados habitats, grupos ecológicos e síndromes de dispersão. Portanto, essas 92 espécies podem ser reagrupadas em comunidades iniciais mais densas e diversas do que as verificadas nos projetos executados. As comunidades vegetais nos locais investigados eram predominantemente compostas por espécies arbóreas e tal padrão pode levar à formação de ecossistemas florestais onde antes havia formações savanânicas.

PALAVRAS-CHAVE: Restauração ecológica. Revegetação. Áreas mineradas. Cerrado. 


\section{REFERENCES}

ANAND, M.; DESROCHERS, R. E. Quantification of restoration success using complex systems concepts and models. Restoration Ecology, Cambridge, v. 12, n.1, p. 117-123, 2004. http://dx.doi.org/10.1111/j.10612971.2004.00281.x

APG III. An update of the Angiosperm Phylogeny Group classification for the orders and families of flowering plants: APG III. Botanical Journal of the Linnean Society, London, v. 161, p. 105-121, 2009.

ARAÚJO, M. S.; REIS, S. F. As implicações ecológicas da variação intrapopulacional. Ciência \& Ambiente, Santa Maria, v. 39, p. 95-108, 2009.

BARBOSA, K. C.; PIZO, M. A. Seed rain and seed limitation in a planted gallery forest in Brazil. Restoration Ecology, Cambridge, v. 14, n. 4, p. 504-515, 2006. http://dx.doi.org/10.1111/j.1526-100X.2006.00162.x

BARBOSA, L. M.; BARBOSA, J. M.; BARBOSA, K. C.; POTOMATI, A.; MARTINS, S. E.; ASPERTI, L. M.; MELO, A. C. G.; CARRASCO, P. G.; CASTANHEIRA, S. A.; PILIACKAS, J. M.; CONTIERI, W. A.; MATTIOLI, D. S.; GUEDES, D. C.; SANTOS-JÚNIOR, N. A.; SILVA, P. M. S.; PLAZA, A. P. Recuperação florestal com espécies nativas no Estado de São Paulo: pesquisas apontam mudanças necessárias. Florestar Estatístico, São Paulo, v. 6, n. 14, p. 28-34, 2003.

BARBOSA, L. M.; BARBOSA, T. C.; BARBOSA, K. C.; PARAJARA, F. C. Práticas e políticas públicas para a restauração ecológica a partir de reflorestamentos com alta diversidade de espécies regionais. In: MARTINS, S. V. (ed.) Restauração ecológica de ecossistemas degradados. Viçosa: Editora UFV, 2012. p. 240-261.

CARVALHO, P. E. R. Espécies arbóreas brasileiras. Brasília: Embrapa Informação Tecnológica, 2008. v. 3, $593 \mathrm{p}$.

CORLETT, R. T.; HAU, B. C. H. Seed dispersal and forest restoration. In: ELLIOTT, S.; KERBY, J.; HARDWICK, K.; BLAKESLEY, D.; WOODS, K.; ANUSARNSUNTHORN, V. (Eds.). Forest restoration for wildlife conservation. Thailand: International Tropical Timber Organisation and The Forest Restoration Research Unit, 2000. p. 317-325.

CORRÊA, R. S. Recuperação de áreas degradadas pela mineração no Cerrado: manual para revegetação. 2 ed. Brasília: Editora Universa, 2009. 174 p.

CORRÊA, R. S.; BIAS, E. S.; BAPTISTA, G. M. M. Áreas degradadas pela mineração no Distrito Federal. In: CORREAA, R. S.; BAPTISTA, G. M. M. (Orgs.). Mineração e áreas degradadas no Cerrado. Brasília: Editora Universa, 2004. p. 9-21.

CORRÊA, R. S.; MÉLO FILHO, B.; BAPTISTA, G. M. M. Avaliação fitossociológica da sucessão autogênica em áreas mineradas no Distrito Federal. Cerne, Lavras, v. 13, n. 4, p. 406-415, 2007.

DAJOZ, R. Ecologia geral. Petrópolis: Editora Vozes \& Editora Universidade de São Paulo, 1983. 480 p.

DAJOZ, R. Princípios de ecologia. 7 ed. Porto Alegre: Artmed, 2005. 474 p.

DUBOC, E. Cultivo de espécies nativas do bioma Cerrado. Comunicado Técnico 110. Planaltina: Empresa Brasileira de Pesquisa Agropecuária, Ministério da Agricultura e Abastecimento, 2004. 10 p.

DUBOC, E.; GUERRINI, I. A. Crescimento inicial e sobrevivência de espécies florestais de matas de galeria no domínio do Cerrado em resposta à fertilização. Energia na Agricultura, Botucatu, v. 22, n.1, p. 42-60, 2007. 
DURIGAN, G. Bases e diretrizes para a restauração da vegetação de Cerrado. In: KAGEYAMA, P. Y.; OLIVEIRA, R. E.; MORAES, L. F. D.; ENGEL, V. L.; GANDARA, F. B. (Orgs.). Restauração ecológica de ecossistemas naturais. Botucatu: Fundação de Estudos e Pesquisas Agrícolas e Florestais - FEPAF, 2003. p. 185-204.

DURIGAN, G.; ENGEL, V. L. Restauração de ecossistemas no Brasil: onde estamos e para onde podemos ir? In: MARTINS, S. V. (Ed.). Restauração ecológica de ecossistemas degradados, Viçosa: Editora UFV, 2012. p. 41-68.

DURIGAN, G.; SILVEIRA, E. R. Recomposição da mata ciliar em domínio de cerrado, Assis, SP. Scientia Forestalis, Piracicaba, v. 56, p. 135-144, 1999.

EITEN, G. Vegetação do Cerrado. In: PINTO, M. T. N. (Org.). Cerrado: caracterização, ocupação e perspectivas. 2 ed. Brasília: Editora Universidade de Brasília, 1994. p. 7-73.

EITEN, G. Vegetação natural do Distrito Federal. Brasília: Editora Universidade de Brasília/SEBRAE-DF, 2001. $162 \mathrm{p}$.

ENGEL, V. L.; PARROTTA, J. A. Definindo a restauração ecológica: tendências e perspectivas mundiais. In: KAGEYAMA, P. Y.; OLIVEIRA, R. E.; MORAES, L. F. D.; ENGEL, V. L.; GANDARA, F. B. (Orgs.). Restauração ecológica de ecossistemas naturais. Botucatu: Fundação de Estudos e Pesquisas Agrícolas e Florestais - FEPAF, 2003. p. 1-26.

GOTELLI, N. J.; COLWELL, R. K. Quantifying biodiversity: procedures and pitfalls in the measurement and comparison of species richness. Ecology Letters, Davis, v. 4, n. 4, p. 379-391, 2001.

GUIMARÃES JR., P. R. A estrutura e a dinâmica evolutiva de redes mutualísticas. Ciência \& Ambiente, Santa Maria, v. 39, p. 137-148, 2009.

INMET. Instituto Nacional de Meteorologia. Available in: $<$ http://www.inmet.gov.br/> Access on August $9^{\text {th }}$ 2012.

IUCN. International Union for Conservation of Nature. Available in:

http://www.iucn.org/about/work/programmes/pa/paproducts/wcpacategories/ Access on December $18^{\text {th }} 2010$.

KAGEYAMA, P. Y.; GANDARA, F. B.; OLIVEIRA, R. E. Biodiversidade e restauração da floresta tropical. In: KAGEYAMA, P. Y.; OLIVEIRA, R. E.; MORAES, L. F. D.; ENGEL, V. L.; GANDARA, F. B. (Orgs.).

Restauração ecológica de ecossistemas naturais. Botucatu: Fundação de Estudos e Pesquisas Agrícolas e Florestais - FEPAF, 2003. p. 27-48.

KLINK, C. A.; MACHADO, R. B. A conservação do Cerrado brasileiro. Megadiversidade, Belo Horizonte, v. 1, n. 1, p. 147-155, 2005.

LYONS, K. G.; BRIGHAM, C. A.; TRAUT, B. A.; SCHWARTZ, M. W. Rare species and ecosystem functioning. Conservation Biology, Cambridge, v. 19, n. 4, p. 1019-1024, 2005.

http://dx.doi.org/10.1111/j.1523-1739.2005.00106.x

MARON, M.; HOBBS, R. Replacing lost ecosystems - the Devil is in the detail. Balancing biodiversity offsets with restoration reality. Decision Point, Melbourne, n. 63, p. 6-7, 2012.

MARTINS, H. F. Floresta da Tijuca. Rio de Janeiro: Centro de Pesquisas e de Conservação da Natureza, 1966. $96 \mathrm{p}$. 
MARTINS, S. V.; MIRANDA NETO, A.; RIBEIRO, T. M. Uma abordagem sobre diversidade e técnicas de restauração ecológica. In: MARTINS, S. V. (Ed.). Restauração ecológica de ecossistemas degradados. Viçosa: Editora UFV, 2012. p. 17-40.

MELO, A. C. G.; DURINGAN, G.; KAWABATA, M. Crescimento e sobrevivência de espécies arbóreas plantadas em áreas de cerrado. In: VILAS BOAS, O.; DURIGAN, G. (Orgs). Pesquisas em conservação e recuperação ambiental no Oeste Paulista: resultados da cooperação Brasil-Japão. Assis: Páginas \& Letras, 2004. p. 315-324.

MENDONÇA, R. C.; FELFILI, J. M.; WALTER, B. M.; SILVA JÚNIOR, M. C.; REZENDE, A. V.; FILGUEIRAS, T. S.; NOGUEIRA, P. E. Flora vascular do cerrado. In: SANO, S. M.; ALMEIDA, S. P. (Eds.). Cerrado, ambiente e flora. Planaltina: Embrapa, 1998. p. 289-556.

MENDONÇA, R. C.; FELFILI, J. M.; WALTER, B. M. T.; SILVA JR., M. C.; REZENDE, A.V.; FILGUEIRAS, T. S.; NOGUEIRA, P. E.; FAGG, C. W. Flora vascular do bioma Cerrado. In: SANO, S. M.; ALMEIDA, S. P.; RIBEIRO, J. F. (Eds.). Cerrado: ecologia e flora. Brasília: Embrapa Informação Tecnológica, 2008a. p. 222-279.

MENDONÇA, R. C.; FELFILI, J. M.; WALTER, B. M. T.; SILVA JR., M. C.; REZENDE, A. V.; FILGUEIRAS, T. S.; NOGUEIRA, P. E.; FAGG, C. W. Flora vascular do bioma Cerrado. In: SANO, S. M.; ALMEIDA, S. P.; RIBEIRO, J. F. (Eds.). Cerrado: ecologia e flora. Brasília: Embrapa Informação Tecnológica, 2008b. p. 417-1279.

MOBOT - Missouri Botanical Garden. W3 Tropicos. Available in http://www.mobot.org/w3T/search/vast.html Access on October $22^{\text {nd }} 2012$.

MOKANY, K.; ASH, J.; ROXBURGH, S. Functional identity is more important than diversity in influencing ecosystem processes in a temperate native grassland. Journal of Ecology, London, v. 96, n. 5, p. 884-893, 2008. http://dx.doi.org/10.1111/j.1365-2745.2008.01395.x

NAEEM, S. Biodiversity and ecosystem functioning in restored ecosystems: extracting principles for a synthetic perspective. In: FALK, D.A.; PLAMER, M. A.; ZEDLER, J. B. (Eds.). Foundations of restoration ecology. Washington: Island Press, 2006. p. 210-237.

PEEL, M. C.; FINLAYSON, B. L.; McMAHON, T. A. Updated world map of the Köppen-Geiger climate classification. Hydrology and Earth System Sciences Discussions, Göttingen, v. 4, p. 439-473, 2007. http://dx.doi.org/10.5194/hessd-4-439-2007

PINHEIRO, C. Q.; CORRÊA, R. S.; SILVEIRA, I. M.; JESUS, R. S.; JORGE, R. R. A. Análise fitossociológica do estrato arbóreo de uma cascalheira revegetada no Distrito Federal. Cerne, Lavras, v. 15, n. 2, p. 205-214, 2009.

REIS, A.; BECHARA, F. C.; ESPINDOLA, M. B.; VIEIRA, N. K.; SOUZA, L. L. Restauração de áreas degradadas: a nucleação como base para incrementar os processos sucessionais. Natureza \& Conservação, Curitiba, v. 1, n. 1, p. 28-36, 2003.

REIS, A.; BECHARA, F. C.; TRÊS, D. R. Nucleation in tropical ecological restoration. Scientia Agricola, Piracicaba, v. 67, n. 2, p. 244-250, 2010.

RODRIGUES, R. R.; LIMA, R. A. F.; GANDOLFI, S.; NAVE, A. G. On the restoration of high diversity forests: 30 years of experience in the Brazilian Atlantic Forest. Biological Conservation, Boston, v. 142, n.6, p. 1242-1251, 2009. http://dx.doi.org/10.1016/j.biocon.2008.12.008

RUIZ-JAEN, M. C.; AIDE, T. M. 2005. Restoration success: how is it being measured? Restoration Ecology, Cambridge, v. 13, n. 3, p. 569-577, 2005. http://dx.doi.org/10.1111/j.1526-100X.2005.00072.x 
SER - Society for Ecological Restoration. The Ser primer on ecological restoration. Tucson: International Science and Policy Working Group, 2004. 15 p.

SILVA, L. C. R.; CORRÊA, R. S. Sobrevivência e crescimento de seis espécies arbóreas submetidas a quatro tratamentos em área minerada no Cerrado. Revista Árvore, Viçosa, v. 32, n. 4, p.731-740, 2008.

SINGH, A. N.; RAGHUBANSHI, A. S.; SINGH, J. S. Plantation as a tool for mine spoil restoration. Current Science, Bangalore, v. 82, n. 12, p. 1436-1441, 2002.

SNEDECOR, G. W.; COCHRAN, W. G. Statistical methods. 8 ed. Ames: Iowa State University Press, 1989. $503 \mathrm{p}$.

STARR, C. R.; CORRÊA, R. S.; FILGUEIRAS, T. S.; HAY, J. D. V.; SANTOS, P. F. Plant colonization in a gravel mine revegetated with Stylosanthes spp. in a Neotropical savanna. Landscape and Ecological Engineering, Tokyo, v. 9, n. 1, p. 189-201. 